\title{
Painful dry socket: an alternative perspective
}

SADJ August 2018, Vol 73 no 7 p456 - p458

E Shevel

\begin{abstract}
A new and effective way of treating painful dry socket is described.
\end{abstract}

\section{INTRODUCTION}

Painful dry socket is an unwelcome complication following tooth extraction, ${ }^{1}$ presenting after approximately $3 \%$ of routine extractions and may occur in up to $30 \%$ of patients following surgical removal of impacted mandibular third molars. ${ }^{2}$

The most common symptom of painful dry socket is a continuous throbbing pain that radiates to the ear and temple. ${ }^{3}$ Classically, this starts one to three days postextraction and may be accompanied by other signs and symptoms such as foul taste and halitosis. ${ }^{4-6}$ The symptoms can persist for up to 10 days after extraction and may include pain so severe that it is not relieved by even the strongest of analgesic medications. ${ }^{6}$

The main focus of treatment is the relief of pain, and current therapies are based on the removal of debris from the socket by irrigation, and the use of analgesic medication. ${ }^{4}$ Intra-socket medicaments may be placed such as antibacterials, topical anaesthetics and obtundents or combinations of all three. ${ }^{4}$ These medications include zinc oxide and eugenol impregnated cotton pellets, ${ }^{7}$ lidocaine ointment, ${ }^{8}$ alvogyl (eugenol, iodoform and butamen), ${ }^{9}$ dentalone, bismuth subnitrate, iodoform paste (BIPP) on ribbon gauze and metronidazole. Some studies have also reported the use of lasers for the treatment of dry socket. ${ }^{9}$ Forty-five percent of patients with painful dry socket require multiple postoperative visits, which are timeconsuming for the practitioner, and which could have significant consequences for the individual patient as well as societal costs including time off work. ${ }^{6,10}$

\section{Current perceptions - true or false?}

Exposed Bone is Painful

Contrary to what is widely believed, exposed bone is not painful.

According to Hansen and Pindborg, a dry socket that has lost its blood clot, and has exposed alveolar bone, is not always painful. They called a dry socket accompanied

Elliot Shevel: BDS, DipMFOS, MBBCh. The Headache Clinic, 45 Empire Rd. Parktown 2193, Johannesburg. Tel: 0114840933. Email: drshevel@theheadacheclinic.net by severe pain "alveolitis sicca dolorosa", or "painful dry socket". They also described a dry socket that is not painful as "alveolitis simplex"11 and has also been called "clinical socket". 12

That exposed bone is not painful is vividly illustrated in a passage from the book "Oral Surgery" by WH Archer, published in 1968. Archer relates the following tale of a patient who had 22 teeth extracted, and was instructed to keep his mouth clean. Three days later, when the patient returned for a post-operative consultation, his mouth was scrupulously clean, and every socket was devoid of a blood clot, as he had used cotton buds to clean them. The patient reported no pain or discomfort of any kind. ${ }^{13}$ This is very easy for the reader to verify. Probing a dry socket with a dental probe is painless, unless there is a loose fragment of bone as a result of fracture during the extraction. If pressure is placed on this fragment there may well be pain because of the impact on the underlying tissues. Touching or probing the solid lamina dura of the socket is, however, painless.

In painful dry socket, the pain comes from the socket. All of the therapies currently in use to treat painful dry socket are designed predominantly to reduce the pain, but an extensive search of the literature on painful dry socket reveals that the literature is devoid of evidence that the origin of the pain is in the socket. All the treatment modalities are designed to treat the pain that is presumed to come from the tooth socket itself, but there is no evidence to show that the pain actually originates in the socket. A most comprehensive and authoritative Cochrane review on dry socket, conducted in 2012, makes no mention of the origin of the pain. ${ }^{14}$

Where then does the pain of dry socket originate? This paper describes a radical departure from the current thinking on dry socket, and describes:

1. The origin of the pain,

2. Alternative and highly efficacious treatment, and

3. Simple preventive precautions which may dramatically reduce the incidence of post-operative pain. This is particularly pertinent in patients having third molar removal.

The author holds the opinion that the pain of dry socket comes predominantly from two places:

1. The periodontal ligament of the tooth adjacent to the extraction site, and 
2. The masseter and at times the temporalis muscles.

Consider the following experiences of the author ;

The periodontal ligament

In every case of painful dry socket, one or both of the teeth adjacent to the extraction socket is or are sensitive to percussion.

This may be confirmed by percussing the teeth with the back of a mouth mirror, starting three or four teeth from the socket, and progressively percussing the teeth towards the affected socket. Invariably, the tooth next to the socket is more sensitive to percussion than are the teeth more distant from the socket.

In the case of painful dry socket after removal of mandibular wisdom teeth, the lower second molars are tender.

\section{The muscles of mastication}

There is tenderness on palpation of the masseter muscles, most commonly at the anterior margin of the masseter where it is attached to the zygoma (Figure 1), but any part of the muscle may be involved.

There may also be tenderness on palpation of the temporalis muscle.

The fact that the masseter and temporalis muscles are painful explains why the most Figure 1: The region of the masseter muscle common symptom of most commonly found to be tender in dry socket is a contin-

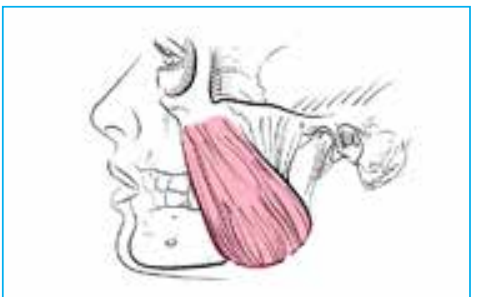

uous throbbing pain that radiates to the ear and temple. ${ }^{3}$ Pain originating in the masseter muscle is known to radiate to the ear.

Why is the periodontal ligament of the adjacent tooth tender to percussion?

The most likely explanation is that following the extraction of surgical removal of a tooth, there is a certain amount of inflammatory oedema in the surrounding tissues. If this inflammatory oedema also affects the periodontal ligament of the adjacent tooth, then the tooth will be slightly extruded, causing a premature occlusion with the opposing tooth, which in turn will lead to sensitivity to percussion of the extruded tooth. The more trauma during the extraction, the more post-operative oedema can be expected and the greater the chance of painful dry socket developing. ${ }^{15}$

\section{Why do the muscles become painful?}

The most likely explanation for this appears to be that it results from the premature occlusion and periodontal pain of the extruded tooth.

\section{TREATMENT}

The treatment of painful dry socket can usually be achieved in a single visit.

If the tooth adjacent to the extraction site is tender to percussion, then a simple bite equilibration results in almost instantaneous relief of the patient's pain.
There are some caveats however:

1. The tenderness of the involved tooth may prevent the use of articulating paper to pinpoint which part of the tooth should be ground down to relieve the prematurity.

2. Hence the suggestion that any equilibration be carried out on the opposing tooth, as the involved tooth may be painful to work on.

Regarding socket debridement, the author does not carry this out in the rooms. The patient is instead provided with a syringe with a curved nozzle that can easily be inserted into the socket, and is instructed to irrigate the socket after meals.

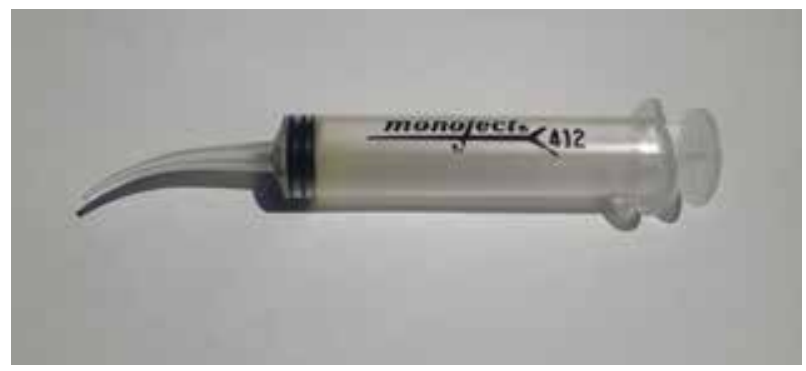

Figure 2: Monoject 412 syringe for socket debridement.

\section{PREVENTION}

The main object of prevention is to obviate post-operative pain.

Prior to extracting a tooth, the adjacent teeth should be percussed to ascertain whether they are at all tender to percussion. If they are, then the bite should be equilibrated prior to the extraction. This will minimise the chances of a dry socket becoming painful.

The author has been using this preventive treatment for forty years, and has found that the incidence of post-operative pain following third molar removal has decreased dramatically.

Clot breakdown and the development of dry socket may still occur. The condition is, however, no longer painful.

\section{DISCUSSION}

The Cochrane Collaboration published a review in 2012 of the local interventions for the management of dry socket. The aim of this systematic review was to analyse the different methods currently used in the management of dry socket..$^{14}$ Although dry socket is one of the most studied complications in dentistry, ${ }^{16}$ and despite the plethora of different methods used in the management of dry socket pain, a further review concluded in 2015 that there was no evidence to support any of the interventions currently in use..$^{17}$ As there have been no recent developments in the management of dry socket since 2015 , this conclusion remains valid to this day.

An earlier study had found that the incidence of painful dry socket following single extractions was $5 \%$, whereas the occurrence of the condition following multiple extractions was $2.1 \% .^{18}$ Statistically, the difference between these proportions is highly significant $(\mathrm{P}<0.001) .{ }^{18}$

Krogh found that "the more adjacent teeth removed at one operation, the less the danger of (painful) dry 
socket development". ${ }^{19}$ This is counter-intuitive. It may be expected that the number of painful dry sockets should increase in linear proportion to the number of teeth removed. However, consider the role of the periodontal membrane in influencing pain. If one tooth is removed, unless it is a third molar, there is usually a tooth on either side of it, which means that there are two teeth adjacent to the single extraction socket, either or both of which may experience periodontal inflammation and contribute to pain. If two adjacent teeth are removed, then there is still a tooth on either side of the gap that may become painful. Hence again only two possibly painful teeth but now per two sockets. Effectively the chances of painful dry socket are halved. If three adjacent teeth are extracted, then there are still only two possibly painful teeth, meaning that the potential incidence of painful dry socket per tooth socket is now further reduced. This explains Krogh's observation that "the more adjacent teeth removed at one operation, the less the danger of dry socket development". ${ }^{19}$

There are observations relevant to and supportive of these concepts:

- The author has never seen a case of painful dry socket following a full dental clearance, even though clot breakdown most certainly occurs in these cases. He has also been unable to find a single report of this in the literature. This supports the understanding that without teeth adjacent to the extraction site, dry sockets, if they do occur, are not painful.

- Dry socket pain also never occurs when fractured mandibles are immobilised, even when teeth in the fracture line have been extracted or teeth have been lost during the trauma. When a fractured mandible is wired in occlusion, the teeth adjacent to the extracted teeth cannot be extruded by periapical oedema, because they are firmly in occlusion. The author has not found it necessary to debride and pack an extraction socket while the jaws are wired in occlusion.

\section{CONCLUSION}

The pain of dry socket results from bite prematurities affecting the tooth or teeth next to the socket. These prematurities are the most likely cause of the muscle pain that develops one to three days post-extraction.

The prevention of post-operative pain is accomplished by equilibration of the bite before undertaking the extraction or surgical removal of a tooth. This is only necessary if the tooth adjacent to the extraction socket is tender to percussion before the extraction. If the teeth adjacent to the extraction are not sensitive to percussion before the extraction, the chances of painful dry socket developing are considerably reduced. On the contrary, if the teeth adjacent to the extraction are sensitive to percussion, the chances of post-extraction pain are greatly enhanced.

The recommended treatment of painful dry socket is to equilibrate the bite by removing the premature contacts of the teeth adjacent to the extraction socket, together with ensuring that the socket is kept free of debris.

\section{Footnote}

The author has practised as a Maxillo-Facial and Oral Surgeon since 1973 and over the years has removed countless impacted wisdom teeth. He has not found it necessary to use Alvogyl or any other socket medication since 1979.

\section{References}

1. Burgoyne CC, Giglio JA, Reese SE, Sima AP, Laskin DM. The efficacy of a topical anesthetic gel in the relief of pain associated with localized alveolar osteitis. J Oral Maxillofac Surg. 2010;68(1):144-8

2. Bowe DC, Rogers S, Stassen LF. The management of dry socket/alveolar osteitis. J Ir Dent Assoc. 2011;57(6):305-10.

3. Swanson AE. A double-blind study on the effectiveness of tetracycline in reducing the incidence of fibrinolytic alveolitis. J Oral Maxillofac Surg. 1989;47(2):165-7.

4. Blum IR. Contemporary views on dry socket (alveolar osteitis): a clinical appraisal of standardization, aetiopathogenesis and management: a critical review. Int J Oral Maxillofac Surg. 2002;31(3):309-17.

5. Noroozi AR, Philbert RF. Modern concepts in understanding and management of the "dry socket" syndrome: comprehensive review of the literature. Oral Surg Oral Med Oral Pathol Oral Radiol Endod. 2009;107(1):30-5.

6. Vezeau PJ. Dental extraction wound management: medicating postextraction sockets. J Oral Maxillofac Surg. 2000;58(5):531-7.

7. Bloomer CR. Alveolar osteitis prevention by immediate placement of medicated packing. Oral Surg Oral Med Oral Pathol Oral Radiol Endod. 2000;90(3):282-4.

8. Silva LJ, Poi WR, Panzarini SR, Rodrigues TS, Simonato LE. Clinical evaluation of an ointment with $10 \%$ metronidazole and $2 \%$ lidocaine in the treatment of alveolitis. Minerva Stomatol. 2006;55(7-8):431-6.

9. Kaya GS, Yapici G, Savas Z, Gungormus M. Comparison of alvogyl, SaliCept patch, and low-level laser therapy in the management of alveolar osteitis. J Oral Maxillofac Surg. 2011;69(6):1571-7.

10. Nusair YM, Younis MH. Prevalence, clinical picture, and risk factors of dry socket in a Jordanian Dental Teaching Center. J Contemp Dent Pract. 2007:8(3):53-63.

11. Hansen EH. Alveolitis sicca dolorosa (dry socket): frequency of occurrence and treatment with trypsin. J Oral Surg Anesth Hosp Dent Serv. 1960;18:409-16.

12. Bernier JL. Management of Oral Disease. St. Louis: C. V. Mosby Co.; 1955.

13. Archer H. Oral Surgery. Philadelphia and London: W. B. Saunders Co.; 1969.

14. Daly B, Sharif MO, Newton T, Jones K, Worthington HV. Local interventions for the management of alveolar osteitis (dry socket). Cochrane Database Syst Rev. 2012;12:CD006968.

15. Nusair YM, Goussous ZM. Quantifying the healing of dry socket using a clinical volumetric method. Oral Surg Oral Med Oral Pathol Oral Radiol Endod. 2006;101(6):e89-95.

16. Cardoso CL, Rodrigues MT, Ferreira Junior O, Garlet GP, de Carvalho PS. Clinical concepts of dry socket. J Oral Maxillofac Surg. 2010;68(8):1922-32.

17. Taberner-Vallverdu M, Nazir M, Sanchez-Garces MA, GayEscoda C. Efficacy of different methods used for dry socket management: A systematic review. Med Oral Patol Oral Cir Bucal. 2015;20(5):e633-9.

18. MacGregor AJ. Aetiology of dry socket: a clinical investigation. Br J Oral Surg. 1968;6(1):49-58.

19. Krogh HW. Incidence of dry socket. Jnl Amer Dent Ass and Dent Cos. 1937;24:1829-36. 